\title{
Preparation of copper sulphide clusters in organic-inorganic composites of Langmuir-Blodgett films of amphiphilic Schiff bases
}

\author{
G HEMAKANTHI, ARUNA DHATHATHREYAN*, T RAMASAMI and \\ D MÖBIUS \\ Chemical Sciences Division, Central Leather Research Institute, Adyar, \\ Chennai 600 020, India \\ Max-Planck-Institut für biophysikalische Chemie, Am Fassberg, 37077 \\ Göttingen, Germany \\ e-mail: adhatha@indiaserver.com
}

MS received 20 October 2000; revised 12 January 2001

\begin{abstract}
Copper sulphide clusters were prepared in Langmuir-Blodgett films of copper complexes of amphiphilic Schiff bases-3,4-dimethoxy-N-benzylidene hexadeylamine (I) and 3,4-dimethoxy-N-benzylidene-4'-(hexadecylamino) benzylamine (II) The clusters obtained were analysed using UV-Vis spectroscopy and optical microscopy. Brewster angle microscopic studies on monolayers of I and II at air/water interface showed formation of needle-like domains which seem to cluster faster in I than in II. Atomic force microscopy (AFM) studies also showed fairly uniform sized clusters in II whereas in the case of $\mathbf{I}$ they seem to show varying sizes. From the results it is concluded that $\pi$-elongation in the polar head groups leads to controlled cluster sizes in compound II as compared to those in compound $\mathbf{I}$.
\end{abstract}

Keywords. Copper sulphide clusters; Langmuir-Blodgett films; organic-inorganic composites; amphiphilic Schiff bases.

\section{Introduction}

Organised molecular films of metal complexes such as metal phthalocyanine, metalporphyrin, ruthenium complexes etc. are currently attracting much interest owing to their fascinating chemical, optical, electrical, thermal and electro-optical properties ${ }^{1-4}$. Generally, there are three ways to fabricate organised molecular films of metal complexes using the Langmuir-Blodgett (LB) technique. The first method is to transfer the monolayer of complexes themselves onto the solid substrate. The second is to spread the amphiphilic organic ligand onto the subphase containing metal ions and form the monolayer of metal complexes in situ. LB films of metal complexes can be fabricated by subsequent deposition of the monolayer. The third method is to fabricate an LB film of the ligand. A subsequent reaction of the ligand LB films with metal ions can also cause the formation of the LB films of the metal complexes. However in order to form metal complexes in monolayers and in LB films, the selection of appropriate ligands is very

*For correspondence 
important. A number of studies on amphiphilic organic ligands and selective complexation of these with transition metal ions have been reported ${ }^{5,6}$.

In recent years, much attention has been paid to the surface modification of inorganic particles with organic functional molecules ${ }^{7-9}$. Such combination offers the possibility of improving the optical, electronic, magnetic and chemical properties of the composite particles to a great extent. Moreover, it also facilitates manipulation of their behaviour and thus provides a way to construct nanomaterials from nanocomponents. Alivisatos et $a l^{10}$ have reported that gold nanoparticles modified with single stranded DNA oligonucleotides can be assembled into dimers and trimers on addition of a complementary single-stranded DNA template. Preparation and characterization of metal nanoclusters using the Langmuir-Blodgett (LB) technique have been widely reported ${ }^{11-15}$. LB films not only offer the possibility of built-in architectural control at the monolayer level, but also provide useful media for the controlled fabrication of quantum sized (Q-sized) particles under mild conditions ${ }^{16-18}$. The formation of inorganic nanometer size semiconductor particles by exposure of fatty acid salt LB films to $\mathrm{H}_{2} \mathrm{~S}$ atmosphere was shown by Smotkin et $a l^{16}$. Some interesting nonlinear phenomena were observed on these particles, such as a blue shift in the optical absorbance ${ }^{16,19}$.

In this work, amphiphilic Schiff bases-3,4-dimethoxy-N-benzylidene hexadeylamine (I) and 3,4-dimethoxy-N-benzylidene-4'-(hexadecylamino) benzylamine (II) were synthesized and were used to form monolayers at the air/water interface as well as in LB films. Complexation of I and II to copper ions from the subphase and subsequent formation of copper sulphide in LB films of copper complexes of I and II were monitored using UV-Vis spectroscopy. Optical microscopy was used to study the $\mathrm{CuS}$ clusters formed in both the matrices.
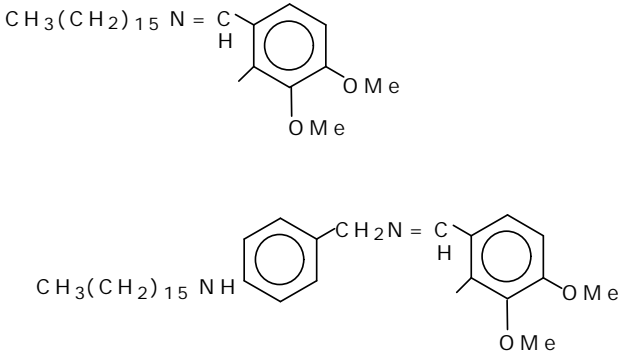

II

Brewster angle microscopy (BAM) was used to analyse the morphology of the monolayers on subphases containing copper ions. Such amphiphilic Schiff bases have been known to form stable monolayers at the air/water interface and can complex with a variety of metal ions ${ }^{20,21}$. The matrix containing the unreacted amphiphilic ligand can be removed selectively providing self aggregation of particles sometimes in the nanometer range. The films thus formed have an average thickness of about $0.6 \mathrm{~nm}$ per LB bilayer of initial amphiphile. Such metal particles passivated by surfactants have been shown to be stable under normal conditions in solution, as films and as dry powders and are dispersible in non-polar solvents ${ }^{22}$. In this work, the effect of the presence of aromatic ring with $o$-methoxy substituents in 3,4-positions and the effect of elongation of the $\pi$ electron system on the clustering and size of the particles of $\mathrm{CuS}$ have been analysed. Monolayer-forming abilities of $\mathbf{I}$ and II at the air/water interface have been studied and 
the surface pressure-area isotherms recorded. Copper complexes formed by spreading the compounds on copper ions have been studied using BAM. The LB films of these complexes have been exposed to $\mathrm{Na}_{2} \mathrm{~S}$ and the formation of $\mathrm{CuS}$ monitored using $\mathrm{UV}$ Vis spectroscopy and optical microscopy.

\section{Experimental}

Hexadecylamine, 4-(hexadecylamino) benzylamine, 3,4-dimethoxy benzaldehyde, sodium sulphide $\left(\mathrm{Na}_{2} \mathrm{~S}\right)$ used for the synthesis were obtained from Sigma Chemicals, USA and were $99 \%$ pure. The solvents used to carry out the reaction (methanol), for recrystallisation and spreading of the monolayers (dichloromethane, HPLC grade) were obtained from E Merck, Germany. Compounds I and II were synthesized by reacting equimolar amounts of the respective amines with 3,4-dimethoxy benzaldehye in methanol and heating the mixture to about $40^{\circ} \mathrm{C}$. The product obtained was recrystallized several times from chloroform:methanol (3:1 $v / v$ ratio) and characterized by gas chromatography, showing $99 \%$ purity, and by elemental analysis, confirming the expected amounts of $\mathrm{C}$ and $\mathrm{H}$ within $0.2 \%$ accuracy. Quartz slides $(30 \times 10 \times 1.5 \mathrm{~mm})$, cleaned thoroughly with chromic acid, were used to transfer the monolayers using the LB technique.

The monolayer experiments were carried out with a computerized NIMA 611 singlebarrier trough. A Wilhelmy balance was used to measure surface pressure to an accuracy of $0.1 \mathrm{mN} / \mathrm{m}$. For all experiments the temperature was kept at $21 \pm 0.5^{\circ} \mathrm{C}$. Clean quartz slides were used to transfer the films at a surface pressure of $25 \mathrm{mN} / \mathrm{m}$. The films transferred were of the X-type and the transfer ratio was nearly 0.9. LB films of copper complexes were subjected to sulphidation (S) by exposing the films to a reaction chamber containing $\mathrm{Na}_{2} \mathrm{~S}$ in isopropyl alcohol. Absorption spectra were recorded on a SHIMADZU UV-160 spectrophotometer. A Zeiss-Jena optical microscope with $\times 10$ and $\times 100$ objectives were used for the optical microscopic studies. Formation of copper sulphide was monitored using UV-Vis spectra and compared with that of the pure metal complex.

The Brewster angle microscopy technique in particular allows phase transitions in molecular films at the liquid/air interface to be studied. In this technique, a parallel light beam polarised in the plane of incidence is reflected at the Brewster angle on the air/water interface. The reflected light is detected by a microscope connected to a video camera. The reflected light is sensitive to local density or thickness differences which appear in the molecular film during compression. A MINI-BAM microscope (Nanofilm technologie, $\mathrm{GmbH}$ ) was used for this measurement ${ }^{23}$. For atomic force microscopy, freshly cleaved mica sheets were used as substrates to transfer the LB films. The samples were imaged with a Nanosope II atomic force microscope (Digital Instruments,Santa Barbara, CA). Silicon nitride tips (Digital Instruments) were used in contact mode. Line scan frequencies were between 2 and $3 \mathrm{~Hz}$. The images were unfiltered except for slope removal along each scan line to level the image.

\section{Results and discussion}

Figures $1 \mathrm{a}$ and $\mathrm{b}$ show the $\pi-A$ isotherm of $\mathbf{I}$ and II spread from dichloromethane solution on pure water surface and aqueous subphases containing $\mathrm{Cu}^{2+}$. By extrapolating the linear part of the $\pi-A$ isotherm to the zero surface pressure, a limiting area of 

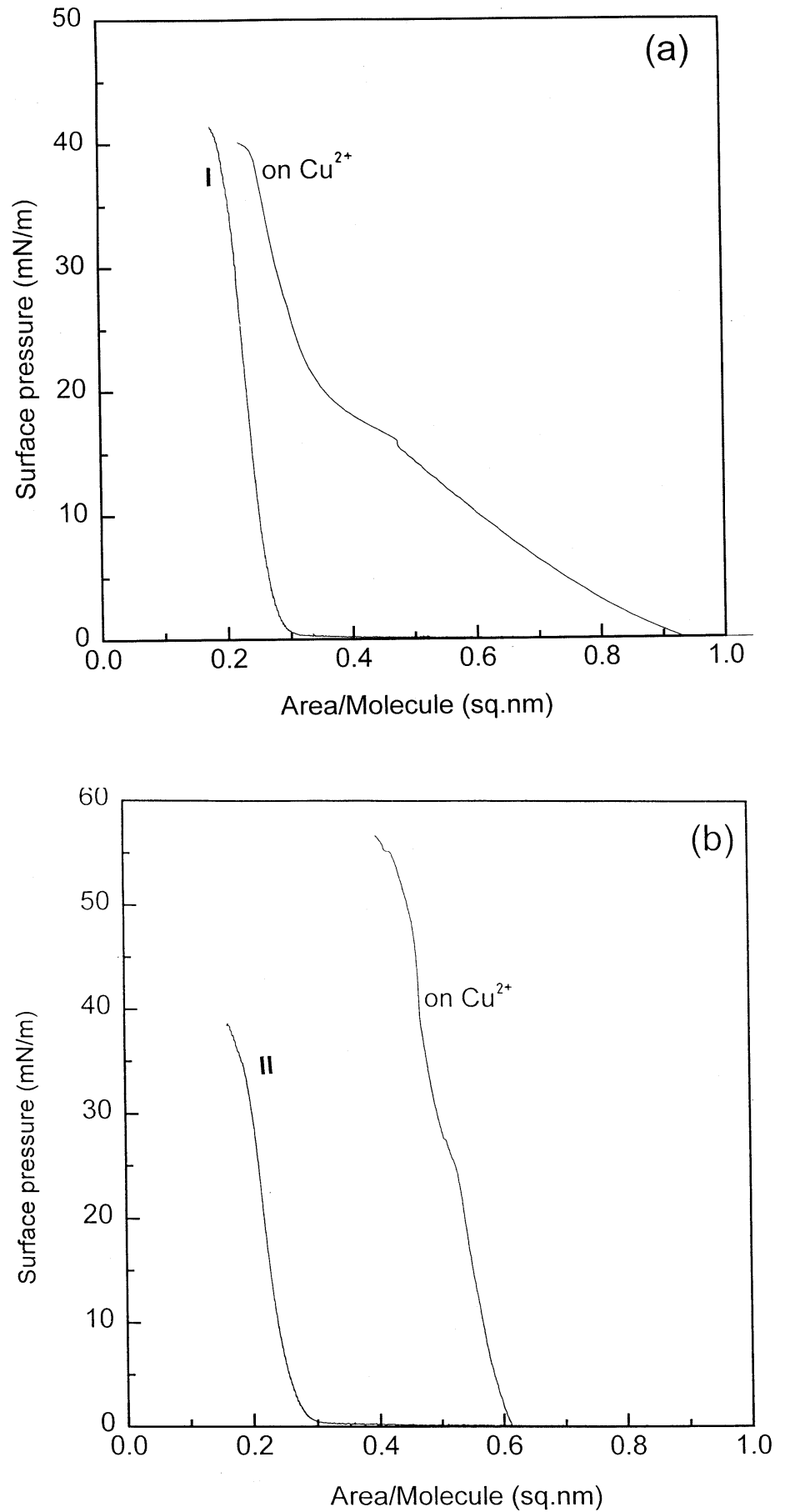

Figure 1. $\pi-A$ isotherms of $\mathbf{I}(\mathbf{a})$ and $\mathbf{I I}$ (b) on deionised distilled water and $\mathrm{Cu}^{2+}$ (concentration $0.5 \mathrm{mM}$ ) containing subphase. 

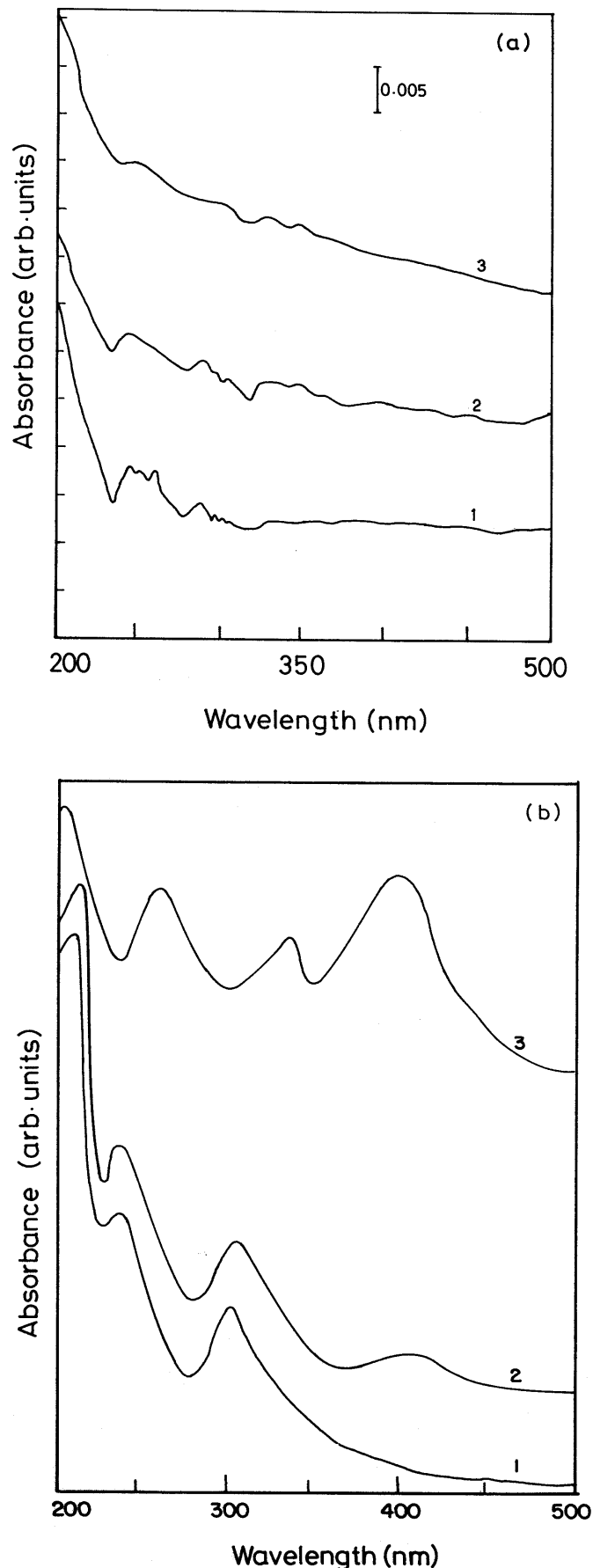

Figure 2. (a) UV-Vis absorption spectra of compound I (1), copper complex of I (2) and CuS (3) in LB film of I. (b) UV-Vis absorption spectra of compound II (1), copper complex of II (2) and CuS (3) in LB film of II. 
$0 \cdot 27 \mathrm{~nm}^{2}$ per molecule is obtained for I and II. The fact that compound II, containing two aromatic rings in the polar plane, still shows the same area as compound I can be explained as follows: Usually the lone pair on the nitrogen atom tends to conjugate with the N-benzyl ring which forces the ring twist and form the non-planar structure as in scheme 1 .

The steric repulsion between the $\mathrm{H}$ atoms of the $-\mathrm{CH}=\mathrm{N}-$ linkage and the $\mathrm{H}$ atoms on the adjacent benzyl ring can also lead to non-planar structure. On the $\mathrm{Cu}^{2+}$ subphase both compounds show expansion indicating strong interaction. From CPK models and the tendency of divalent $\mathrm{Cu}^{2+}$ atoms to assemble ligands to dimers and to form bridging chelating complexes, it is seen that both the ligands form dimeric complexes and this is substantiated by the increase in area of the complexes as compared to that the free ligands. In order to elucidate these changes in the $\pi-A$ isotherms, absorption spectra of LB films transferred at $25 \mathrm{mN} / \mathrm{m}$ were recorded. Figures $2 \mathrm{a}$ and $\mathrm{b}$ show the absorption

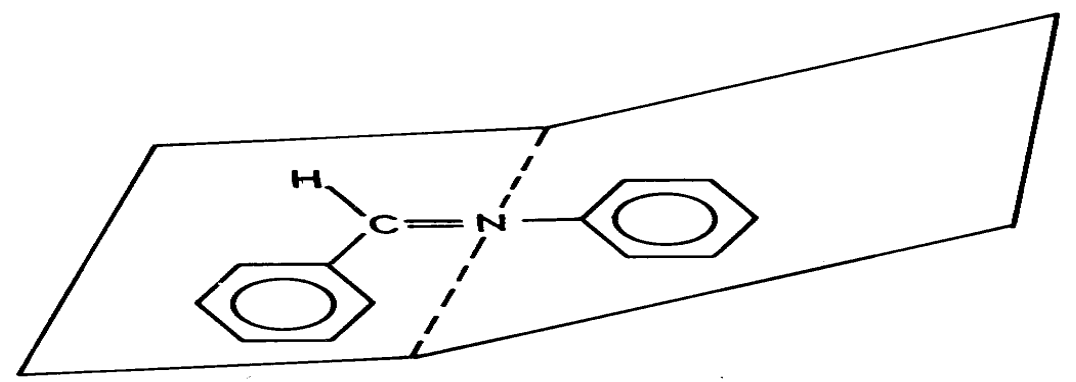

Scheme 1.
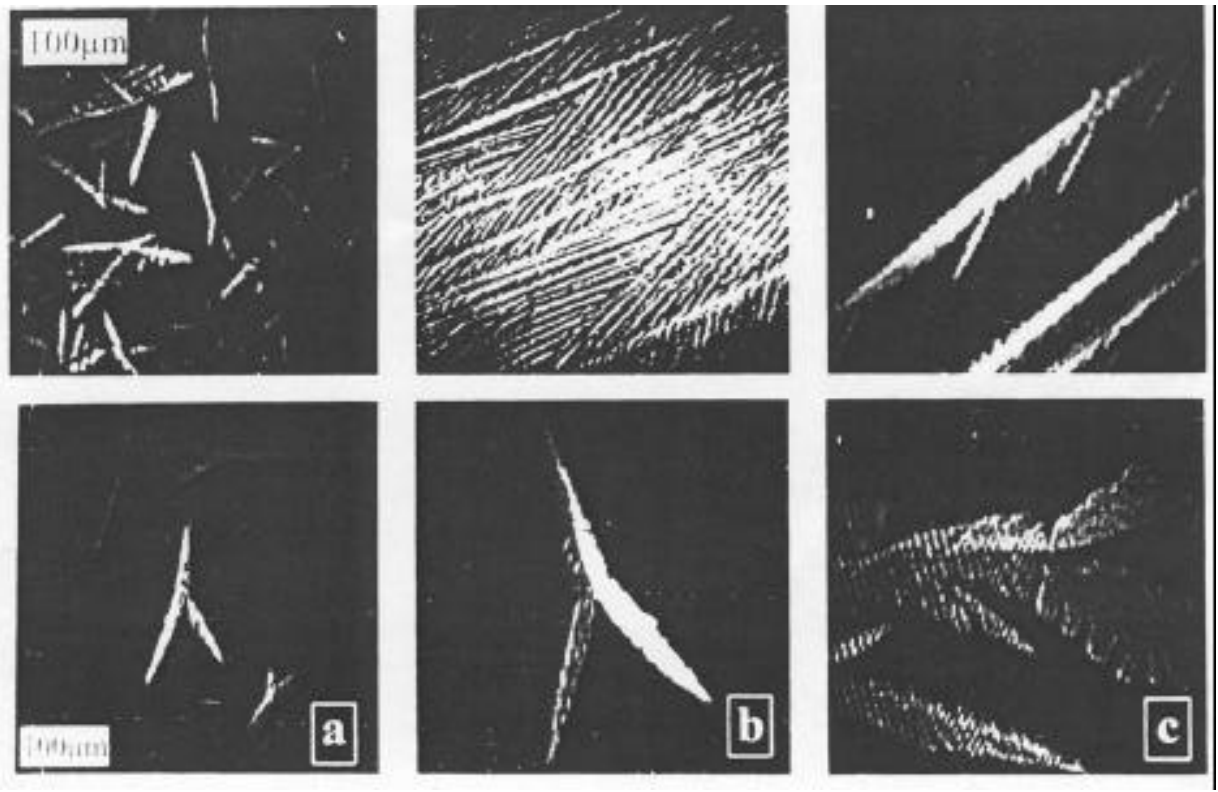

Figure 3. BAM images of compounds $\mathbf{I}$ and $\mathbf{I I}$ on $\mathrm{Cu}^{2+}$ subphase. 

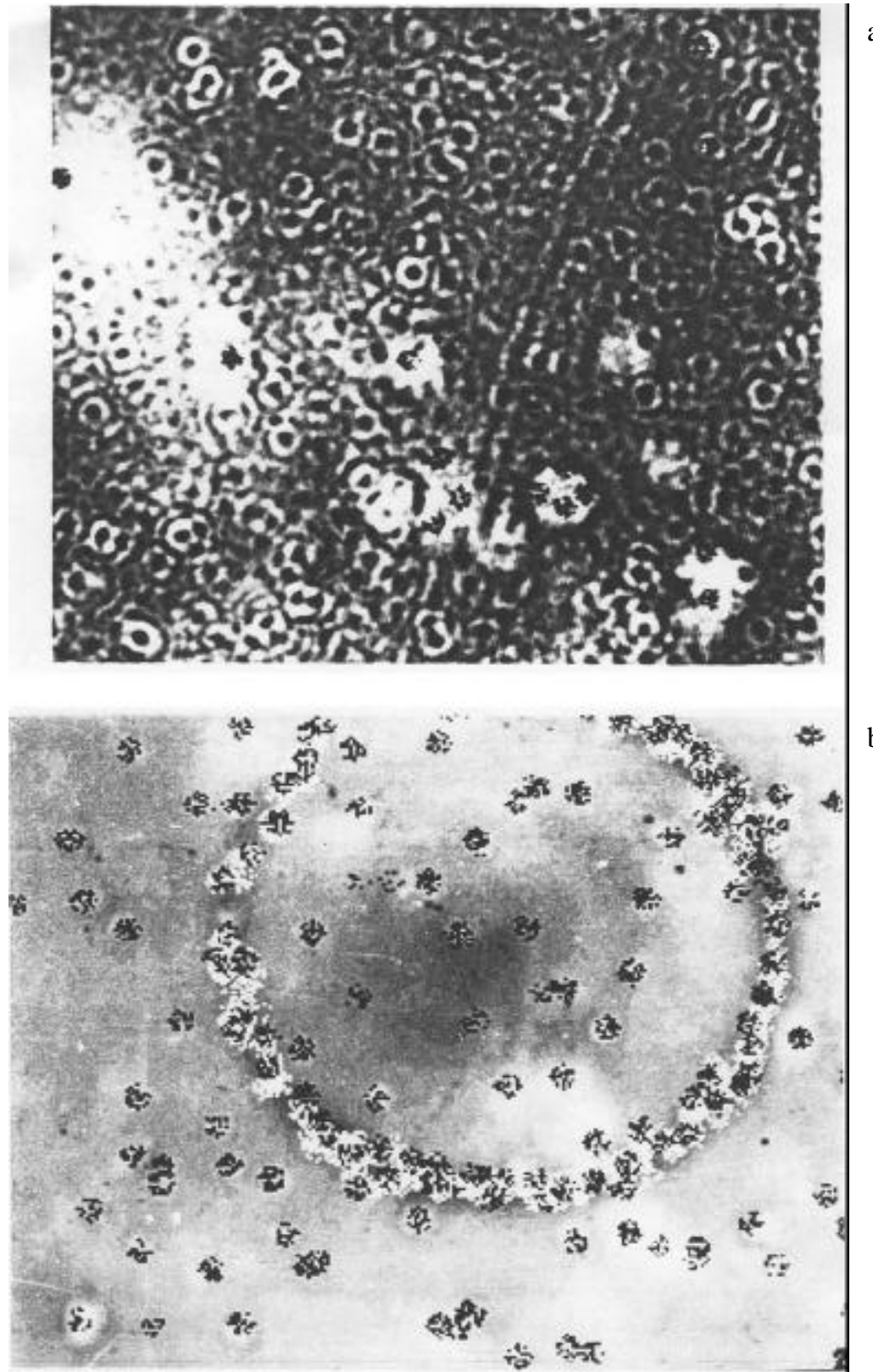

Figure 4. Optical micrographs of $\mathrm{CuS}$ in $\mathrm{LB}$ film of compound I (a) and II (b) (scale: $1 \mathrm{~cm}=10 \mu \mathrm{m}$ ).

spectra of I and II and their copper complexes. Absorption bands are observed between 400 and $450 \mathrm{~nm}$ which correspond to chelating complexes of I and II. The build up of intensity of the band at about $400 \mathrm{~nm}$ in the complex compared to that in the free ligand is indicative of the greater degree of $\pi$-delocalisations in the dimer. The spectra of the corresponding copper sulphide complexes formed are also shown in these figures. 


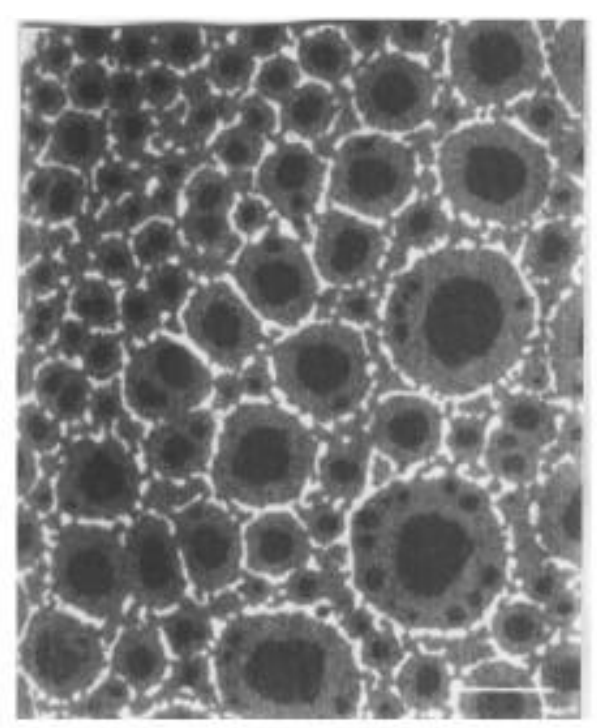

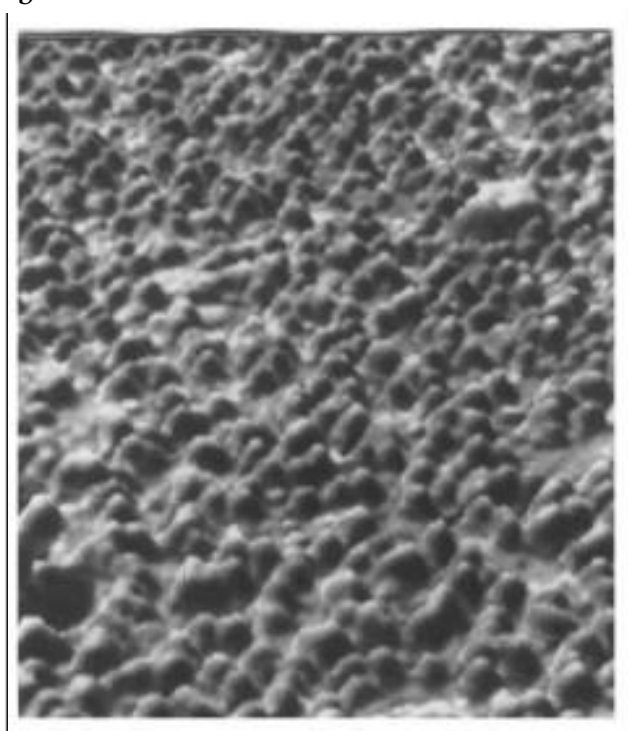

Figure 5. Atomic force microscopic images of $\mathrm{CuS}$ in LB film of compound $\mathbf{I}$ (a) and II (b) (scale: $1 \mathrm{~cm}=10 \mathrm{~nm})$.

$\mathrm{CuS}$ particles generated in the matrix of the amphiphiles in LB films can be described as 3- $d$ quantum wells. The motion of electrons, holes or excitons in such a well is limited and leads to quantization of kinetic energy. In such particles, light absorption corresponds to electron transitions between levels of size-quantization for electrons $E_{\ln }^{e}$ and holes $E_{\text {ln }}^{h}$ with $l, n=$ constant. The position of the absorption band can then be written ${ }^{24}$ as

$$
h v_{01}=E g+\left(h^{2} / 8 \pi^{2} \mu a^{2}\right) J_{\ln }^{2},
$$

where $\mu=m_{e} m_{h} /\left(m_{e}+m_{h}\right)$ and $a$ is the size of the particle. Here the $J$ values are the roots of the Bessel functions with $l$ and $n$ being the angular and spin quantum numbers. The optical absorption edge of such semiconductor particles shows a blue shift as the particle dimensions decrease. In the present work, the size of the CuS particles estimated from the dependence of the absorption edge lies in the range of 3-10 $\mathrm{nm}$.

Inspection of the monolayers by Brewster angle microscopy (BAM) reveal that $\mathbf{I}$ and II are fairly homogenous at the air/water interface. When spread on subphase containing $\mathrm{Cu}^{2+}$ ions, formation of small non-fluid needle-like domains are seen. Compound II seems to form complexes with less clustering. On further compression they coalesce to larger ones. This could mean formation of three-dimensional crystals at the interface (figure 3). LB films of the sulphides of these copper complexes on solid substrates were analysed using optical microscopy. Figures $4 \mathrm{a}$ and $\mathrm{b}$ show the optical micrographs of $\mathrm{CuS}$ in I and II. Both the matrices show fairly homogenous distribution of uniform sized clusters of $\mathrm{CuS}$. Here again $\mathrm{CuS}$ in II matrix seems to show less clustering compared to I as the organization and orientation of the head groups is greatly influenced by the $\pi-\pi$ interactions. AFM pictures of $\mathrm{CuS}$ in matrix I and II in figure 5 show fairly uniform sized clusters for II (around $4 \mathrm{~nm}$ ) whereas I shows flattened structures of varying sizes. 
In conclusion, the amphiphilic Schiff bases synthesized in this study demonstrate that $\pi-\pi$ interactions influence the organization and orientation of the head group. The presence of two aromatic rings in the polar plane of II participate in complex formation leading to less clustering of the copper complex. This extended $\pi$-electron benzilidine compound seems to hinder clustering of the $\mathrm{CuS}$ and leads to fairly uniform and homogenous particle sizes.

\section{Acknowledgements}

One of the authors GH would like to thank Council of Scientific \& Industrial Research, New Delhi for a fellowship. AD thanks Council of Scientific \& Industrial Research, New Delhi, for Raman Research Fellowship, 1999 which funded her stay and work at MPI für Biophysikalische Chemie, Am Fassberg.

\section{References}

1. Schick G A, Schreiman I C, Wagner R W, Lndsy J S and Bocian F 1988 J. Am. Chem. Soc. 1111344

2. Fujiki M and Tabei H 1988 Langmuir 4320

3. Fujihara M, Nishiyama K and Aoki K 1988 Thin Solid Films 160317

4. Nakamura T, Tanaka H and Matsumoto M 1988 Chem. Lett. 1667

5. Liu M, Ushida K, Kira A and Nakahara H 1997 J. Phys. Chem. B101 1101

6. Liu M, Ushida K, Kira A and Nakahara H 1998 Thin Solid Films 327-329 491

7. Murray C B, Kagan C R and Bawendi M G 1995 Science 27024

8. Yoneyama $\mathrm{H}$ and Torimoto T 1995 Adv. Mater. 7492

9. Zhang X, Du S, Chen Y, Zhang L, Cao Y, Chai X, Bai Y, Xiao L and Li T 1998 Thin Solid Films 327-329 563

10. Alivisatos A P, Hohnson K P, Peng X. G, Wilson T E, Loweth C J, Bruchez M P Jr and Schultz P G 1996 Nature (London) 382609

11. Yamaki T, Yamada T, Asai K and Ishigura K 1998 Thin Solid Films 327-329 586

12. Moriguchi I, Matuso K, Sakai M, Hanai K, Teraoka Y and Kagawa S 1998 J. Chem. Soc., Faraday Trans 942199

13. Hemakanthi G and Dhathathreyan A 1999 Langmuir 153317

14. Furlong D N, Urqhart R, Grieser F, Tanaka K and Okahata Y 1993 J. Chem. Soc., Faraday Trans 892031

15. Moruguchi I, Nii H, Nanai K, Nagoka H, Teraska Y and Kagawa S 1995 Colloids Surf. A103 173

16. Smotkin E S, Lee C, Bard A J, Campion A, Fox A, Mallouk T E, Webber S E and White J M 1988 Chem. Phys. Lett. 15265

17. Moruguchi I, Tanaka I, Teraoka Y and Kagawa S 1991 J. Chem. Soc., Chem. Commun. 1401

18. Fendler J H 1995 J. Phys. Chem. 1005500

19. Facci P, Erokhin V, Carrara S and Nicholini C 1996 Proc. Natl. Acad. Sci. USA 9310556

20. Facci P, Erokhin V, Tronin A and Nicholini C 1994 J. Phys. Chem. 9813323

21. Shyamalasundari S, Dhathathreyan A, Kanthimathi M and Nair B U 1997 Langmuir 134923

22. Abe K, Hanada T, Yoshida Y, Tanigaki N, Takiguchi H, Nagasawa H, Nakamoto M, Nagasawa H, Yamaguchi T and Yase K 1998 Thin Solid Fllms 327-329 524

23. Hönig D and Möbius D 1991 J. Phys. Chem. 954590

24. Kulish N R, Kunetz U P and Lisitsa M P 1990 Ukr. Fiz. Zh. (Russ. Ed) 351817 